\title{
In vitro antibacterial activities of ethanolic extracted leaves of Thevetia peruviana (Pers.) K.Schum [Thevetia Yellow]
}

\author{
Dr. Krati Ghavri ${ }^{1}$, Dr. Madhavi Adhav ${ }^{2}$ \\ ${ }^{1,2}$ Department of Botany, P.M.B. Gujarati Science College, Indore, Madhya Pradesh, India
}

\begin{abstract}
World is endowed with a rich wealth of medicinal plants. The studied plant Thevetia peruviana (Pers.) K. Schum [Thevetia Yellow] is also one of the important medicinal as well as ornamental plant (Apocynaceae) commonly known as yellow oleander. The leaves of Thevetia Yellow were extracted with $95 \%$ ethanol and in aqueous by using Soxhlet Extraction Apparatus. The antibacterial activity was performed against gram positive bacteria Bacillus subtilis and Staphylococcus aureus and gram negative bacteria viz. Escherichia coli, Klebsiella pneumoniae, Proteus vulgaris, Pseudomonas aeruginosa and Salmonella typhi. The result showed that ethanolic and aqueous leaf extract of Thevetia Yellow, having strong antibacterial activities against the entire tested gram positive and gram negative bacteria. The phytochemical studies reveals the presence of alkaloids, essential oils, flavanoids, cardiac glycosides, phenolic compounds, phytosterols, saponins, tannins and terpenoids, which have proven to be potential antibacterial agents and medicinal utility of the plant. Thus Thevetia Yellow leaves may be utilized in the preparation of some newer antibiotics against tested bacteria.
\end{abstract}

Key words: Antibacterial activity, Cardiac glycosides, Phytochemical analysis, Thevetia peruviana (Pers.) K. Schum.

\section{INTRODUCTION}

Nature is a source of medicinal agents for thousands of year and an impressive number of modern drugs have been isolated from natural sources. According to (WHO) more than $80 \%$ of the world's population relies on traditional medicine for their primary healthcare needs.

Thevetia peruviana (Pers.) K.Schum is a small tree, 15-20 ft. high belongs to the family Apocynaceae originally a native of America and West Indies.Leaves are simple, linear - lanceolate, whorled . Flowers medium, yellow, solitary or in few flowered cymes. (Fig No. 1).All parts of this plant abound in a milky juice which is highly poisonous. (Chopra et al.,1984). The plant is bitter, pungent, acrid, astringent to the bowels, useful in urethral discharges , worms, skin diseases, leucoderma ,wound piles ,eye trouble, itching ,fever and bronchitis.(Kirtikar and Basu,1981 ). The leaves are emetic and purgative, leaf decoction is given to prevent conception. (Ambasta, 1986; Kaushik and Diman ,1999; Retnam and Martin, 2006). The cardiac glycosides obtained from bark, kernals and flowers are useful for heart diseases (Prajapati e .al., 2007).

\section{MATERIAL AND METHODS}

\section{1 .Collection of Plant Material}

Plant materials (ThevetiaYellow Leaves) of Thevetia peruviana (Pers.) K. Schum were collected from Devi Ahilya Vishwavidyalaya campus, Indore. The collected plant materials were identified with the help of Flora of Madhya Pradesh. (Mudgal et al.,1997).

\section{Extraction}

To obtain ethanolic extract $100 \mathrm{gm}$. of shade dried plant material was extracted with $500 \mathrm{ml}$. of ethanol $(95 \%)$ in "Soxhlet Extraction Apparatus. Finally the prepared plant material was macerated with water for 24 hrs. to obtain aqueous extract. Each extract was concentrated by distilling off the solvent (Kokate, 1994 and Kokate et al.,1993).

\section{Preliminary Phytochemical Screening}

The extract thus obtained was than subjected to preliminary phytochemical screening for identification of various plant constituents by methods suggested by (Finar,1962 ; Farnsworth, 1966; Harborne,1973; Harborne et al.,1979).

\section{Antibacterial Testing}

Each extract sample was tested for antibacterial activity against human pathogenic bacteria by 'Cup Borer Method (Kavanagh, 1963; Cheesbrough, 1993). The cultures of bacteria have been obtained from Microbial Type Culture, Gene Bank Chandigarh. 
DOI: 10.17148/IARJSET.2021.81218

The name and culture number of bacteria are as follows:-

Gram positive bacteria

Bacillus subtilis ATCC 6633 and Staphylococcus aureus ATCC 9144

Gram negative bacteria

Escherichia coli. MTCC 739, Klebsiella pneumoniae ATCC 33495,Salmonella typhi ATCC 10749,Pseudomonas aeruginosa ATCC 25668,Proteus vulgaris MTCC 1771

\section{OBSERVATIONS AND DISCUSSION}

\section{Phytochemical screening}

The leaf extract of Thevetia Yellow reveals the presence of alkaloids, flavanoids, glycosides-cardiac glycosides, phenolic compounds, tannins, phytosterols, carbohydrates, saponins, proteins and amino acids was noted in the observation Table, while fixed oils, fats, gums and mucilages were found absent. (Table No. 01).

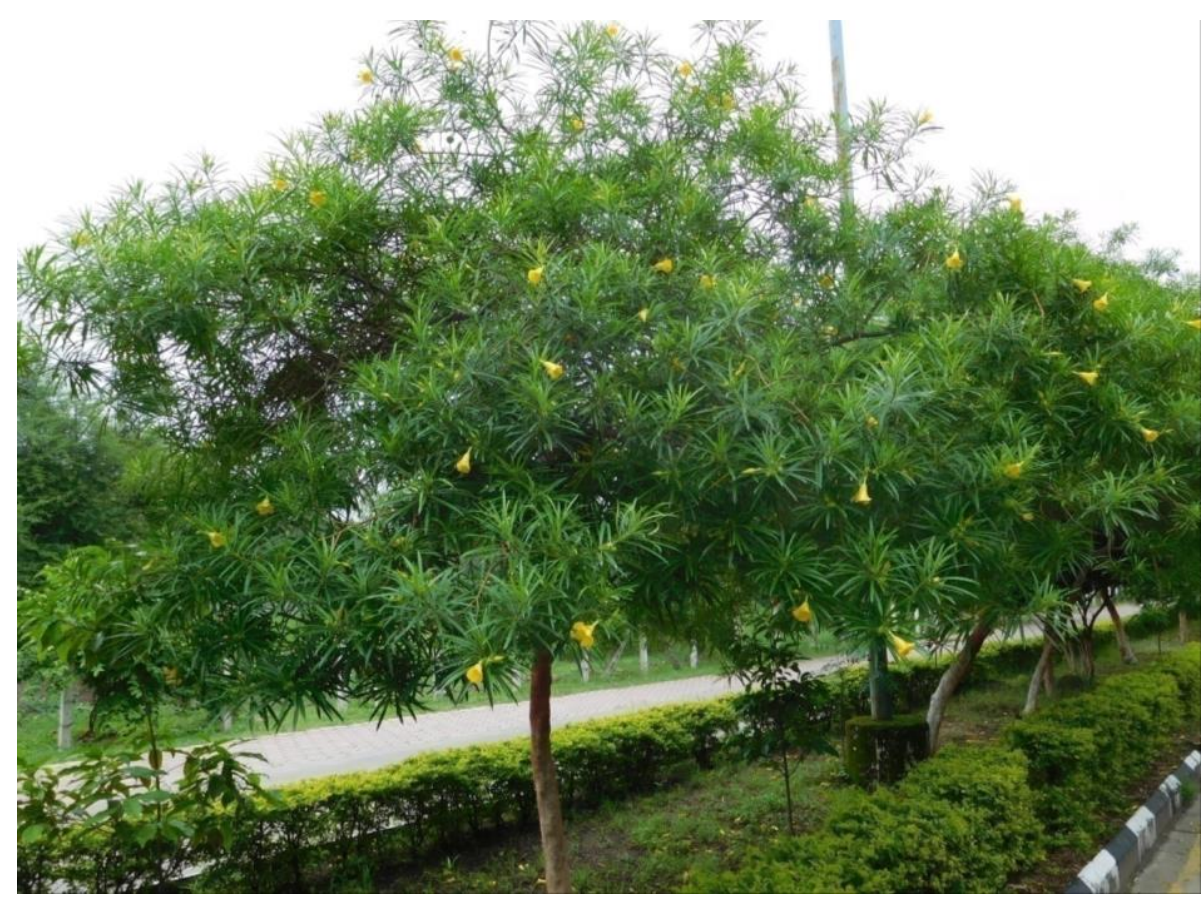

Fig.No. 1: Thevetia peruviana (Pers.) K. Schum [ThevetiaYellow]

\section{Antibacterial Testing}

The ethanolic and aqueous leaf extracts of Thevetia Yellow exhibits strong antibacterial activity against Bacillus subtilis , Staphylococcus aureus, Escherichia coli, Klebsiella pneumoniae, Proteus vulgaris, Pseudomonas aeruginosa and Salmonella typhi.

Table 1: Preliminary phytochemical screening of ethanolic leaf extract of Thevetia peruviana (Pers.) K.Schum [Thevetia Yellow]

\begin{tabular}{|l|l|l|}
\hline S. No. & Plant Constituents Test/Reagents & Result \\
\hline 1. & Alkaloids & \\
\hline & Mayer's reagent & + \\
\hline & Dragendorff's reagent & + \\
\hline & Hager's reagent & + \\
\hline & Wagner's reagent & + \\
\hline 2. & Carbohydrates & + \\
\hline & Molish's reagent & + \\
\hline & Benedict's reagent & + \\
\hline & Fehling solution & \\
\hline 3. & Types of Carbohydrates & \\
\hline
\end{tabular}


International Advanced Research Journal in Science, Engineering and Technology

Vol. 8, Issue 12, December 2021

DOI: $10.17148 /$ IARJSET.2021.81218

\begin{tabular}{|c|c|c|}
\hline & Glucose & + \\
\hline & Fructose & + \\
\hline & Galactose & - \\
\hline & Lactose & + \\
\hline & Starch & + \\
\hline \multirow[t]{2}{*}{4.} & Phytosterols & \\
\hline & Liebermann-Burchard's test & + \\
\hline \multirow[t]{2}{*}{5.} & Terpenoids & \\
\hline & Salkowski reaction & + \\
\hline \multirow[t]{2}{*}{6.} & Fixed oils and fats & \\
\hline & Spot test & - \\
\hline \multirow[t]{2}{*}{7.} & Saponins & \\
\hline & Foam test & + \\
\hline \multirow[t]{2}{*}{8.} & Phenolic compounds & \\
\hline & Ferric chloride solution & + \\
\hline \multirow[t]{2}{*}{9.} & Tannins & \\
\hline & Lead acetate solution & + \\
\hline \multirow[t]{3}{*}{10.} & Proteins & \\
\hline & Biuret test & + \\
\hline & Xanthoprotic test & + \\
\hline \multirow[t]{2}{*}{11.} & Amino acids & \\
\hline & Ninhydrin reagent & + \\
\hline \multirow[t]{2}{*}{12.} & Gums and mucilages & \\
\hline & Alcoholic precipitation & - \\
\hline \multirow[t]{3}{*}{13.} & Flavanoids & \\
\hline & Shinoda test & + \\
\hline & Lead acetate test & + \\
\hline \multirow[t]{2}{*}{14.} & Cardiac glycosides & \\
\hline & Killer kiliani test & + \\
\hline
\end{tabular}

$+=$ Present,$-=$ Absent

Fig.No.2: Antibacterial activity of ethanolic leaf extract of Thevetia peruviana (Pers.) K.Schum [ThevetiaYellow] against gram positive and gram negative bacteria.

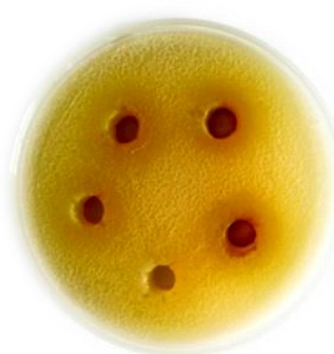

Bacillus subtilis

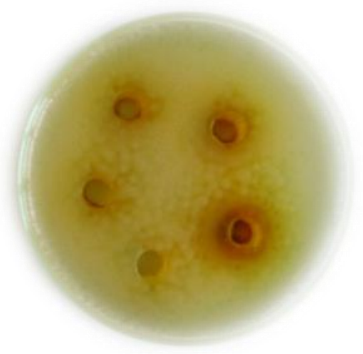

Proteus vulgaris

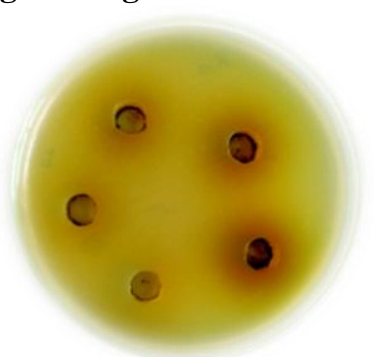

Staphylococcus aureus

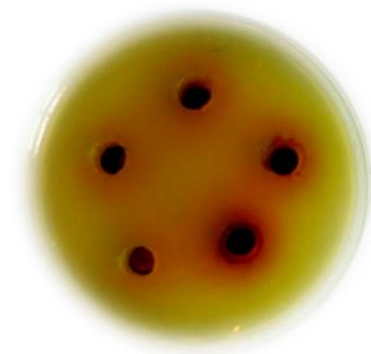

Pseudomonas aeruginosa

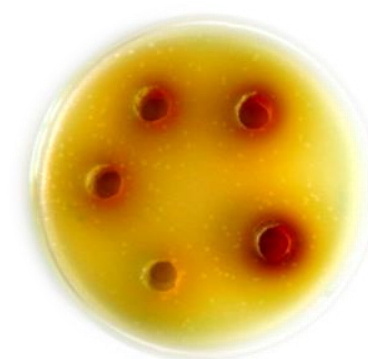

Escherichia coli Klebsiella pneumonia

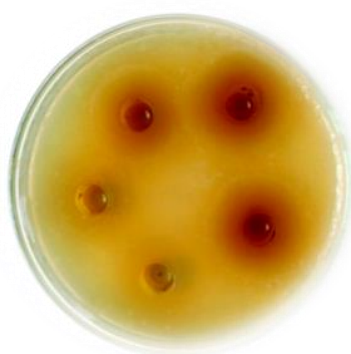

Salmonella typhi 
International Advanced Research Journal in Science, Engineering and Technology

Vol. 8, Issue 12, December 2021

DOI: 10.17148/IARJSET.2021.81218

Fig.No.3: Antibacterial activity of leaf (aqueous extract) of Thevetia peruviana (Pers.) K.Schum [Thevetia Yellow] against gram positive and gram negative bacteria.

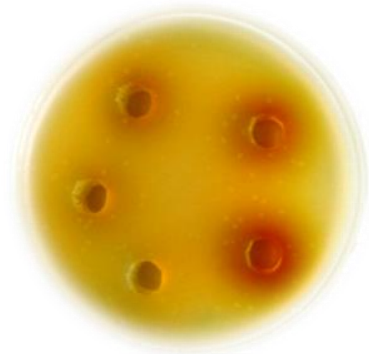

Bacillus subtilis

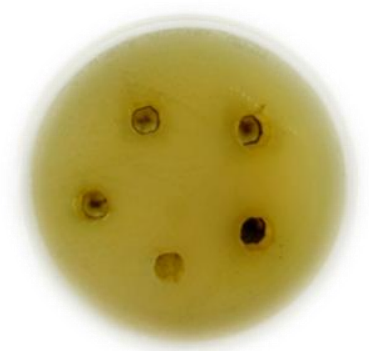

Proteus vulgaris

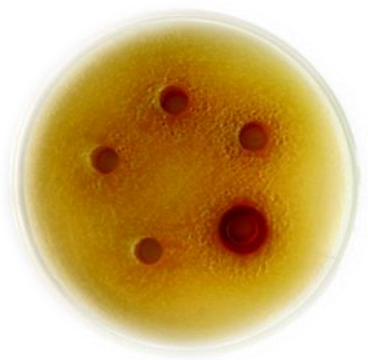

Staphylococcus aureus

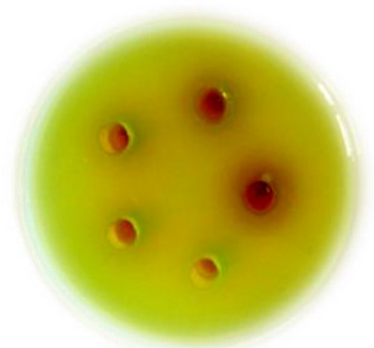

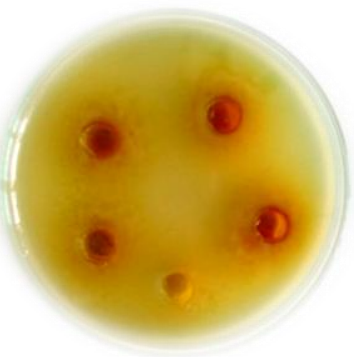

Escherichia coli

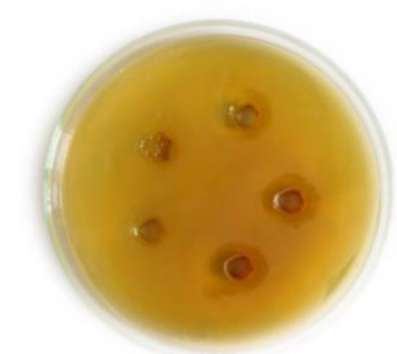

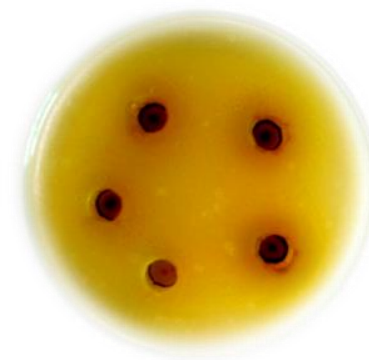

Klebsiella pneumonia

Table No. 2: Antibacterial activity of leaf extracts (ethanolic and aqueous) of Thevetia Yellow against gram positive and gram negative bacteria

\begin{tabular}{|c|c|c|c|c|c|c|c|c|c|}
\hline & & & & & & & & & \\
\hline \multirow{2}{*}{$\begin{array}{l}\text { S. } \\
\text { N } \\
\text { o. }\end{array}$} & \multirow[t]{2}{*}{$\begin{array}{l}\text { Extract } \\
\text { used }\end{array}$} & \multirow{2}{*}{$\begin{array}{l}\text { Quanti } \\
\text { ty } \\
\text { of } \\
\text { extract } \\
\text { in ml. }\end{array}$} & \multicolumn{2}{|c|}{$\begin{array}{l}\text { Gram positive } \\
\text { bacteria }\end{array}$} & \multicolumn{5}{|c|}{ Gram negative bacteria } \\
\hline & & & $\begin{array}{l}\text { Bacillu } \\
\text { S } \\
\text { subtili } \\
\text { S }\end{array}$ & $\begin{array}{l}\text { Staphyloco } \\
\text { ccus aureus }\end{array}$ & $\begin{array}{l}\text { Escheric } \\
\text { hia coli. }\end{array}$ & $\begin{array}{l}\text { Klebsie } \\
\text { lla } \\
\text { pneum } \\
\text { onia }\end{array}$ & $\begin{array}{l}\text { Proteu } \\
\text { S } \\
\text { vulgar } \\
\text { is }\end{array}$ & $\begin{array}{l}\text { Pseudom } \\
\text { onas } \\
\text { aeruginos } \\
\text { a }\end{array}$ & $\begin{array}{l}\text { Salmon } \\
\text { ella } \\
\text { typhi }\end{array}$ \\
\hline & & & \multicolumn{7}{|c|}{ Average diameter of zone of inhibition in $\mathbf{m m}$. } \\
\hline \multirow{6}{*}{1 . } & \multirow{5}{*}{$\begin{array}{l}\text { Ethano } \\
\text { lic }\end{array}$} & .05 & $\begin{array}{l}\text { No } \\
\text { Zone }\end{array}$ & No Zone & No Zone & $\begin{array}{l}\text { No } \\
\text { Zone }\end{array}$ & $\begin{array}{l}\text { No } \\
\text { Zone }\end{array}$ & 10 & $\begin{array}{l}\text { No } \\
\text { Zone }\end{array}$ \\
\hline & & .08 & 12 & 12 & No Zone & 12 & $\begin{array}{l}\text { No } \\
\text { Zone }\end{array}$ & 12 & $\begin{array}{l}\text { No } \\
\text { Zone }\end{array}$ \\
\hline & & .11 & 13 & 13 & 12 & 14 & $\begin{array}{l}\text { No } \\
\text { Zone }\end{array}$ & 13 & 16 \\
\hline & & .14 & 14 & 14 & 14 & 16 & 10 & 14 & 18 \\
\hline & & .17 & 16 & 16 & 16 & 18 & 12 & 16 & 20 \\
\hline & \multicolumn{2}{|l|}{$\mathbf{r}$} & 0.85 & 0.85 & $\mathbf{0 . 9 3 3}$ & 0.894 & 0.886 & 0.990 & 0.921 \\
\hline \multirow{6}{*}{2.} & \multirow{5}{*}{$\begin{array}{l}\text { Aqueo } \\
\text { us }\end{array}$} & .05 & $\begin{array}{l}\text { No } \\
\text { Zone }\end{array}$ & No Zone & No Zone & $\begin{array}{l}\text { No } \\
\text { Zone }\end{array}$ & $\begin{array}{l}\text { No } \\
\text { Zone }\end{array}$ & No Zone & $\begin{array}{l}\text { No } \\
\text { Zone }\end{array}$ \\
\hline & & .08 & $\begin{array}{l}\text { No } \\
\text { Zone }\end{array}$ & No Zone & No Zone & $\begin{array}{l}\text { No } \\
\text { Zone }\end{array}$ & $\begin{array}{l}\text { No } \\
\text { Zone }\end{array}$ & No Zone & $\begin{array}{l}\text { No } \\
\text { Zone }\end{array}$ \\
\hline & & .11 & 12 & No Zone & No Zone & 12 & $\begin{array}{l}\text { No } \\
\text { Zone }\end{array}$ & No Zone & 10 \\
\hline & & .14 & 14 & No Zone & 8 & 13 & $\begin{array}{l}\text { No } \\
\text { Zone }\end{array}$ & 11 & 11 \\
\hline & & .17 & 16 & 14 & 10 & 14 & 8 & 13 & 12 \\
\hline & \multicolumn{2}{|l|}{$\mathbf{r}$} & 0.933 & 0.707 & 0.889 & 0.906 & 0.707 & 0.885 & 0.912 \\
\hline
\end{tabular}

\section{$\mathbf{r}=$ Correlation coefficient}

Pseudomonas aeruginosa

Salmonella typhi

$r=+1$ perfect positive correlation , $r=-1$ perfect negative correlation 


\section{CONCLUSION}

It was concluded that the leaves of selected medicinal plant Thevetia peruviana (Pers.) K. Schum [Thevetia Yellow] is a source of various phytochemicals viz. alkaloids, essential oils, flavanoids, cardiac glycosides, phenolic compounds, phytosterols, saponins, tannins and terpenoids.The antibacterial testing result showed that ethanolic and aqueous leaf extract of Thevetia Yellow, having strong antibacterial activities against gram positive bacteria Bacillus subtilis and Staphylococcus aureus and gram negative bacteria viz. Escherichia coli, Klebsiella pneumoniae, Proteus vulgaris, Pseudomonas aeruginosa and Salmonella typhi. The presence of different phytochemicals proves its correlation with antibacterial activity. Thus Thevetia Yellow leaves may be useful for the formulation of some newer antibiotics against tested bacteria for treating various diseases.

\section{ACKNOWLEDGEMENT}

We wish to express our sincere gratitude to the Principal and Head of the Department of Botany, P. M .B. Gujarati Science College, Indore, for provided full research laboratory facilities throughout our work.

\section{REFERENCES}

1. Ambasta, S.P. (1986).The useful plants of India. Publications and Information Directorate, CSIR, New Delhi, India.636.

2. Cheesbrough, M. (1993). Medical laboratory manual for tropical countries. Vol. II .Microbiology EIBS with Tropical Health Technology/Butte worth -Heinemann. Great Britain at the university press, Cambridge, 201.

3. Chopra , S.R.N.; Badhwar, R. L. and Ghosh ,S. (1984).Poisonous Plants of India. Academic Publishers,Jaipur, India .1:665-668.

4. Emiru, Y.K.; Siraj, E.A.; Teklehaimanot, T.T. and Amare, G.G. (2019). Antibacterial Potential of Aloe weloensis (Aloeacea). Leaf Latex against Gram-Positive and Gram-Negative Bacteria Strains. International Journal of Microbiology. 1-4.

5. Farnsworth.(1966).Biological and Phytochemical Screening of Plants. J. pharm. Sci. 55:225-276.

6. Finar ,L .L. (1962).Organic chemistry, Lonngman, Green Grosvent Street, London.

7. Harborne, J.B. (1973). Phytochemical methods, Chapman and Hall Ltd London.49-188.

8. Harborne,J.B.; Mabry,T.J.; Mabry, H. (1979).The flavonoids .Chapman and Hall International Edition, London.

9. Kaushik, P. and Dhiman, A.K.(1999).Medicinal plants and raw drugs of India. Bishen Singh Mahendra pal Singh publication, Dehradun, India. 352-353.

10. Kavanagh, F. (1963).Analytical Microbiology. Academic Press, London, 125-141.

11. Kirtikar,K.R.and Basu,B.D.(1981).Indian medicinal plants. International book distributors.2: 1553-1556.

12. Kokate, C.K;Purohit.A.P.;Gokhale,B.B.(1993). Pharmacognosy, Twelth Edition, Nirali prakashan, Pune,India.90-93.

13. Kokate,C.K. (1994).Practical pharmacognosy ,Fourth Edition, Vallabh prakashan, Delhi,India.107-111.

14. Manandhar, S.; Luitel, S. and Dahal, R.K.(2019). In vitro antimicrobial activity of some medicinal plants against human pathogenic bacteria. Journal of Tropical Medicine, 1-5.

15. Manske, R.H.F. (1960).The Alkaloids. Chemistry and Physiology Academic Press Inc. London, Ltd.7.

16. Mudgal,V.;Khanna,K.K.;Hajra,P.K.(1997) Flora of Madhya Pradesh. The director, Botanical Survey of India, Calcutta.59-60.

17. Prajapati,N.D.;Purohit,S.S.;Sharma,A.K.;Kumar,T.(2007).A Handbook of medicinal plants.Agrobios,Jodhpur,India.511-512.

18. Retnam; Raveendra, K.;Martin ,P. (2006) Ethnomedicinal plants. Agrobios publication, India.

19. Samell ,K.;Sonita,L.;Chanseiha,N; Sokhany,L.;Kosalvorlak,C.; Huykhim,U.; Juthamart, M.; Yaowared ,C.;and Sin,C.(2018).Phytochemical Analysis and Antioxidant Property of Selected Medicinal Plants Native to Cambodia.Drug. Des.Int.Prop.Int.J.1 (2).

20. Chittima Managit, Hiroaki Sakurai And Ikuo Saiki.(2017). Ethanolic extract of Thevetia peruviana flowers enhances TNF- $\alpha$ and TRAIL-induced apoptosis of human cervical cancer cells via intrinsic and extrinsic pathways. oncology letters, 13: 2791-2798. 